\title{
Influence du premier cycle de sélection récurrente appliquée à une population de blé tendre d'hiver
}

\author{
P Brabant 1, J Kervella 2, G Doussinault 3, E Picard 1, M Rousset 4 \\ avec la collaboration technique de $\mathrm{H} \mathrm{Corti}^{1}$ et JP Meunier ${ }^{1}$ \\ 1 CNRS-INRA-UPS, station de génétique végétale, ferme du Moulon, 91190 Gif-sur-Yvette; \\ 2 INRA, station de recherche fruitière méditerranéenne, domaine de Saint-Paul, 84140 Montfavet; \\ 3 INRA, centre de Rennes, station d'amélioration des plantes, BP 29, 35650 Le Rheu; \\ ${ }^{4}$ INRA, station d'amélioration des plantes, domaine de Crouelle, 63039 Clermont-Ferrand Cedex, France
}

(Reçu le 10 septembre 1990; accepté le 15 avril 1991)

\begin{abstract}
Résumé - Une sélection récurrente multicaractère, multilocale et à cycle court est appliquée à une population de blé tendre d'hiver. L'influence du premier cycle de sélection a été jugée en comparant 76 familles $S_{7}$ extraites de la population issue de l'intercroisement de 16 lignées fondatrices, avec 78 familles $S_{5}$ extraites de la population issue de l'intercroisement des familles sélectionnées au premier cycle. Les caractères étudiés sont le rendement et certaines de ses composantes, des caractères liés au développement (date d'épiaison et hauteur) et des facteurs de stabilité du rendement (résistance à la verse, résistance à l'oïdium). La sélection pratiquée a permis un progrès sur la résistance à l'oïdium et sur le poids de $\mathbf{1 0 0}$ grains. Pour le rendement, il n'y a pas eu de gain, la distribution de ce caractère dans la population d'origine présente une dissymétrie droite qui disparaît dans la population issue du premier cycle. Les variances génétiques estimées pour les caractères soumis à la sélection restent stables ou diminuent. La corrélation génétique entre hauteur et rendement est la seule faisant intervenir le rendement qui soit modifiée. Les caractères entre lesquels les corrélations génétiques sont le plus modifiées sont : la hauteur, la date d'épiaison, la largeur de la feuille de l'épi et le nombre d'épillets par épi. Les corrélations environnementales sont stables.
\end{abstract}

blé / sélection récurrente / sélection multicaractère / corrélation

Summary - Effect of the first cycle of recurrent selection on a wheat population. A multitrait, multilocal and short cycle recurrent selection (RS) procedure was used to improve the agronomic value of a population of winter wheat. The influence of the first RS cycle was evaluated through comparison of inbred families issued from the interbreeding of 16 founder lines and the population obtained after the first cycle. Seventy-six $S_{7}$ families represented the original population and $78 S_{5}$ the selected population. Observed traits were yield and some of its components (mean kernel weight, number of spikelets per spike, weight of 5 spikes), earliness, plant height, width and length of flag leaf, lodging and powdery mildew tolerance. An improvement was obtained for mean kernel weight and powdery mildew tolerance but no improvement was observed for yield (table 1). Distribution of this last trait was skewed in the original population and became normal after the first RS cycle (fig 1). Genetic variances of selected traits remained stable or diminished after the first cycle (table II). Some genetic correlations with yield were not modified, except for plant height (table III). Genetic correlations involving plant height, earliness, width and length of flag leaf and number of spikelets per spike were modified. Environmental correlations were stable (table III).

wheat / recurrent selection / multitrait selection / correlation 


\section{INTRODUCTION}

L'idée d'améliorer des populations de maiis, en faisant se succéder des cycles courts constitués de la sélection et de l'intercroisement des individus sélectionnés, a été introduite au début du siècle aux États-Unis. C'est Hull (1945) qui le premier a employé le terme de sélection récurrente pour décrire ce procédé d'amélioration des populations. Gallais (1977) a depuis généralisé et apporté les éléments théoriques pour optimiser la sélection récurrente sur toutes les espèces, quel que soit leur régime de reproduction. Un exemple de réflexion sur l'optimisation globale est donné par Gallais (1988) pour les espèces où l'haplodiploïdisation est facilement utilisable. L'utilisation massive de I'haplodiploïdisation n'est malheureusement pas toujours possible. Chez certaines espèces, elle peut être remplacée par l'utilisation de la single seed descent (SSD) (Goulden, 1939, Fouilloux et Bannerot, 1988) en générations accélérées. Lorsque chez une autogame, pour des raisons techniques, il n'est pas possible d'utiliser l'une ou l'autre des 2 méthodes précédentes, la sélection récurrente à cycle court n'est possible qu'en sélectionnant sur des générations pas ou peu fixées; plantes $S_{0}$, familles $S_{1}$ ou $S_{2}$. Dans ce cas, l'optimisation du schéma général pose de nombreux problèmes. L'approche théorique en terme de génétique quantitative est déjà bien développée (Gallais, 1970, 1979) mais n'apporte que peu de réponses pratiques si les 5 composantes de la variance génétique (en régime consanguin) sont inconnues. Les contraintes biologiques et expérimentales peuvent de surcroît empêcher la mise en place des schémas théoriquement optimaux. L'approche expérimentale est donc tout à fait nécessaire pour identifier, à chaque étape d'un programme de sélection récurrente à cycle court, les facteurs susceptibles de limiter le progrès génétique. Les estimations a posteriori des conséquences de certaines actions de sélection sont aussi un élément essentiel pour ajuster et modifier les schémas utilisés.

Les 2 stations de génétique et d'amélioration des plantes de l'INRA de Clermont-Ferrand et de Rennes et la station de génétique végétale du Moulon travaillent en commun depuis 1978 sur un programme de sélection récurrente appliqué au blé tendre d'hiver. Le schéma retenu est à cycle court de 3 ans. Après l'intercroisement, la sélection est faite sur plantes $S_{0}$ et l'année suivante sur les familles $S_{1}$ issues des meilleures $S_{0}$. Elle est combinée individu-famille. Lors du premier cycle, 1550 plantes $S_{0}$ ont été observées et après autofécondation et sélection, 80 familles $S_{1}$ ont été retenues pour l'intercroisement. Le programme est décrit en détail par Thomas (1986), Kervella (1987) et plus succinctement par Kervella et al (1988), Rousset et al (1988) et Brabant et al (1989). Son objectif est d'améliorer la valeur variétale en lignées (Gallais, 1979) de la population. La sélection pratiquée est multicaractère et multilocale. Les caractères pris en compte portent sur la productivité, la résistance aux ma-ladies et la qualité boulangère. C'est un index phénotypique (Thomas, 1986) qui a permis de choisir les candidats à l'intercroisement. La construction de l'index et les pondérations utilisées ont été déterminées empiriquement. Le nombre très important de caractères soumis à la sélection et le manque d'expérience de la sélection récurrente sur le blé tendre d'hiver a motivé une étude comparative visant à décrire la variabilité disponible chez les parents fondateurs, dans la population d'origine $\left(\mathrm{PA}_{0}\right)$ issue de l'intercroisement des fondateurs et dans la population $\left(\mathrm{PA}_{1}\right)$ issue du premier cycle de sélection (Thomas, 1986). Deux autres études ont été menées pour comparer $\mathrm{PA}_{0}$ et $\mathrm{PA}_{1}$ sur un échantillon aléatoire de lignées obtenues par SSD. La première a été réalisée à Clermont-Ferrand pendant les saisons 1984/1985 et 1985/1986 (Kervella, 1987; Kervella et al, 1988; Rousset et al, 1988). La seconde a été réalisée au Moulon en 1986/ 1987, sur un échantillon de lignées différent de celui utilisé à Clermont-Ferrand. Les résultats obtenus au Moulon seront présentés ici et discutés en fonction des résultats déjà obtenus.

\section{MATÉRIEL ET MÉTHODES}

\section{Matériel végétal}

Pour juger de l'évolution de la valeur variétale d'une population menée en sélection récurrente, il est nécessaire d'estimer les changements introduits (par un ou plusieurs cycles) sur les moyennes, les variances et les corrélations entre les caractères que l'on souhaite améliorer. Les estimations doivent être faites sur des génotypes représentatifs des variétés qu'on développera. Dans notre cas, nous avons utilisé des familles consanguines extraites par SSD de $\mathrm{PA}_{0}$ et de $\mathrm{PA}_{1}$. En 1985/1986, nous avons multiplié au Moulon 160 familles $S_{7}$ de $P A_{0}$ et 160 familles $S_{5}$ de $P A_{1}$. Nous considérerons le résidu d'hétérozygotie et la différence de fixation entre $P A_{0}$ et $P A_{1}$ comme négligeables. Dans ce matériel, il existait beaucoup de lignées très hautes (de taille supérieure à $1,10 \mathrm{~m}$ ) qu'il 
était impossible de juger en culture intensive. Dans chaque population, nous avons retenu pour les expérimentations de la saison suivante, 80 lignées de taille inférieure à $1,10 \mathrm{~m}$ par tirage aléatoire. Les lignées SSD de $\mathrm{PA}_{0}$ provenaient pour moitié de ClermontFerrand, mais seulement 16 de ces lignées étaient communes avec l'expérimentation menée l'année précédente dans cette station. Pour $\mathrm{PA}_{1}$ toutes les lignées provenaient de la station du Moulon.

\section{Dispositif expérimental}

Le fait d'implanter dans des parcelles contiguës des lignées de tailles très différentes augmente la compétition entre génotypes. L'influence de cette compétition sur l'estimation des différents paramètres qui nous intéressent n'est pas connue. Nous savons, par contre, que les terrains d'expérience de la station du Moulon sont d'une grande homogénéité. Les expérimentations en parcelles de $6 \mathrm{~m}^{2}$, menées depuis plusieurs années, n'ont presque jamais fait apparaître d'effets blocs et les coefficients de variation résiduels sur le rendement sont toujours faibles, de l'ordre de $5 \%$. En conséquence, nous avons préféré séparer les lignées en 2 groupes sur le critère de taille et implanter 2 essais. Le premier avec les 40 lignées les plus courtes de chaque population et le second avec les 40 lignées les plus hautes de chaque population. Les 2 essais ont été placés sur 2 parcelles adjacentes du terrain d'expérience et traités de façon strictement identique. Les 4 variétés Camp-Remy, Fidel, Pernel et Promentin ont été utilisées comme témoins. Dans chaque essai, les 84 génotypes (40 lignées) de $\mathrm{PA}_{0}, 40$ lignées de $\mathrm{PA}_{1}$ et 4 témoins) ont été semés dans un dispositf à 2 blocs complets randomisés. La parcelle élémentaire comportait 3 rangs de $2,10 \mathrm{~m}$ de long, espacés entre eux de $0,23 \mathrm{~m}$. Le semis a été effectué le 3 novembre 1986 à une densité de 250 grains au $\mathrm{m}^{2}$. Les conditions de culture utilisées correspondraient à une conduite intensive, toutefois aucun raccourcisseur n'a été employé.

Les 160 lignées ont aussi été semées dans un dispositif qui diffère du précédent par la taille de la parcelle élémentaire qui est monorang. Sur ce dispositif, aucun traitement fongicide n'a été appliqué et la fumure azotée a été plus faible que sur l'essai décrit précédemment. L'objectif de cette pépinière était de juger des comportements vis-à-vis des différentes maladies. La réduction de fumure azotée diminue le risque de verse qui complique la prise de notation et modifie le développement des maladies. Seul l'oïdium (Erysiphe graminis L) s'est bien développé au Moulon en 1987.

\section{Caractères mesurés}

Sur le dispositif en culture intensive les caractères suivants ont été mesurés :

- P15 poids de grains récolté sur la parcelle et ramené à $15 \%$ d'humidité, exprimé en g;
- PCG poids de 100 grains exprimé en g, calculé à partir du nombre de grains dans un échantillon de 20 g;

- P5E poids de 5 épis bien formés non battus, exprimé en g;

- HAU hauteur en centimètres, mesurée au sommet de l'épi;

- VER verse, notée de 1 (absence) à 9 (totalement couché);

- DEP date d'épiaison mesurée en nombre de j après le premier mai;

- LOF longueur de la feuille de l'épi en $\mathrm{mm}$, moyennes sur 5 talles bien formées;

- LAF largeur de la feuille de l'épi en mm, moyenne sur 5 talles bien formées;

- NEE nombre d'épillets par épi, moyenne sur 5 épis.

Les attaques d'oïdium, OID (Erysiphe graminis L) ont été notées suivant une échelle de 1 (absence d'attaque) à 5 (plante très attaquée).

À l'exception de DEP, $L O F$ et $L A F$, tous les caractères ont été soumis à sélection pour passer de $\mathrm{PA}_{0}$ à $\mathrm{PA}_{1}$ (Thomas, 1986). Pour la hauteur, la sélection a consisté dans le rejet des extrêmes.

\section{Analyses statistiques}

Sur les témoins, le modèle fixe d'analyse de variance suivant :

$$
Y_{i j k}=M u+e s s_{i}+t e m_{j}+e s s \times t e m_{i j}+b l c / e s s_{i k}+R_{i j k}
$$

nous a permis de juger de l'homogénéité du dispositif expérimental.

ess : effet du facteur "essai», à 2 niveaux; tem : effet du facteur "témoin", à 4 niveaux; ess $x$ tem : effet de l'interaction entre les 2 facteurs précédents; blc/ess : effet du facteur «bloc» hiérarchisé au facteur essai; à 2 niveaux par essai.

L'analyse de variance sur les témoins ne fait apparaître un effet essai que sur 3 variables : $L O F, L A F$, NEE. Pour ces 3 variables, les probabilités associées au «F» de Fisher sont $0,028,0,025$ et 0,023 respectivement. L'interaction entre témoin et essai n'est jamais significative au seuil de 0,05 , l'effet bloc dans essai n'est pas davantage significatif au seuil de 0,05. Le coefficient de variation résiduel obtenu sur les témoins pour $\mathrm{P} 15$ est de $0,108\left(M u=1306 \mathrm{~g}, s_{R}=141\right.$ g).

Sur les lignées de chaque population les distributions des moyennes sur les 2 blocs ont été étudiées pour chaque caractère. Les tests de normalité ont été faits sur les coefficients d'asymétrie et d'aplatissement de Pearson. Les comparaisons de distributions entre $P A_{0}$ et $P A_{1}$ ont été réalisées avec le test de Kolmogorov-Smirnov. Les comparaisons de moyennes entre populations ont été faites par un test de Student, unilatéral pour tous les caractères ayant subi une sélection directionnelle, bilatéral pour les autres caractères. 
La variance résiduelle employée pour réaliser les tests est issue de l'analyse de variance faite avec le modèle :

$$
Y_{i j k}=M u+e s s_{i}+p o p_{j}+b l c / e s s_{i k}+R_{i j k}
$$

La définition des différents facteurs est la même que précédemment; $p o p$ désigne le facteur population à 2 niveaux

Pour l'étude de l'évolution des variances et des corrélations entre caractères, nous avons déclaré le facteur "lignée" aléatoire. La structuration en 2 essais ne permettait a priori qu'un traitement essai par essai, il existe en effet une confusion entre l'effet "essai" et l'effet «hauteur des lignées". Les traitements préliminaires effectués sur les témoins ont justifié un regroupement des 2 essais pour une analyse unique. Ceci nous a conduit à estimer les carrés (CM) et produits moyens du facteur lignée, population par population, à partir du modèle suivant:

$$
Y_{i j}=M u+L i g_{i}+R_{i j}
$$

Les carrés et produits moyens résiduels ont par contre été estimés, population par population, à partir du modèle :

$$
Y_{i j k}=M u+e s s_{i}+\text { Lig/ess }_{i j}+b l c / e s s_{i k}+E_{i j k}
$$

Les estimations des carrés et produits moyens "lignée" obtenues grâce au premier modèle et les estimations des carrés et produits moyens résiduels obtenus dans le second sont (en l'absence d'effet «essaj» dû au terrain d'expérience) les plus proches de ceux qu'on aurait obtenus dans une expérimentation où les lignées n'auraient pas été séparées sur la taille. Pour nous assurer de la stabilité des résultats obtenus, nous avons aussi traité des données sur les lignées avec un modèle unique et en déclarant le facteur "essai" (résultats non reproduits). Des effets "essai" significatifs apparaissent sur la plupart des variables. Toutefois, les modifications introduites dans les estimations de variances et de corrélations sont tout à fait mineures et ne changent pas l'interprétation.

La variance du facteur «lignée» que nous appellerons par la suite variance génétique, a été estimée par 1/2(CM $\times$ Lig - CM $\times E)$. La variance environnementale est directement estimée par $C M \times E$. Les estimations des covariances ont été réalisées de la même façon mais à partir des produits moyens. Les héritabilités au sens large ont été estimées par le rapport des estimées de la variance génétique et de la somme des variances génétique et environnementale.

La comparaison des coefficients de corrélation génétique pose des problèmes mal résolus statistiquement. En particulier, la distribution de l'estimateur du coefficient de corrélation génétique est inconnue (Becker, 1984). En conséquence, étant donné la difficulté à estimer les écarts types des erreurs faites sur les estimations des coefficients de corrélation génétique, nous nous sommes fixés arbitrairement les règles de décision suivantes:
- on considère qu'il y a modification de corrélation dès que la valeur absolue de la différence entre les coefficients de corrélation estimés dans chaque population dépasse 0,20 ;

- pour les coefficients de corrélation n'entrant pas dans la catégorie précédente, on considère que les corrélations sont stables et différentes de 0 si la plus petite des 2 estimations dépasse 0,20 en valeur absolue.

Les analyses statistiques ont été faites grâce au logiciel STAT-ITCF version 4 (ITCF Boigneville 91720 Maisse), la programmathèque AMANCE (Bachacou et al, 1981) et le logiciel MODL! (INRA Biométrie Versailles).

\section{RÉSULTATS}

Après élimination des lignées pour lesquelles il existait des données manquantes dans les 2 blocs de l'essai intensif, il restait 76 lignées de $\mathrm{PA}_{0}$ et 78 de $\mathrm{PA}_{1}$. Dans l'essai en pépinière les données concernant les 80 lignées de chaque population étaient toutes disponibles.

L'étude des distributions des moyennes (sur les 2 blocs) des lignées, population par population, montre sur les coefficients d'asymétrie et d'aplatissement (fig 1) des écarts à la normalité pour P15, HAU, VER dans $\mathrm{PA}_{0}$ et pour $H A U$, VER et $O I D$ dans $\mathrm{PA}_{1}$. P15 dans $\mathrm{PA}_{0}$ et $O I D$ dans $\mathrm{PA}_{1}$ présentent une dissymétrie droite (médiane à droite de la moyenne). La comparaison des distributions entre $\mathrm{PA}_{0}$ et $\mathrm{PA}_{1}$ par le test de Kolmogorov-Smirnov ne révèle de différence significative $(p=6 \%$ ) que pour la notation d'oïdium.

L'analyse de variance sur les 8 variables mesurées dans l'essai intensif ne révèle pas d'effet significatif du facteur population. Pour les caractères soumis à sélection, le test de Student unilatéral montre qu'un progrès a été réalisé (tableau 1) pour le poids de 100 grains $(\rho<5 \%$ ) et la résistance à l'oïdium $(p<1 \%)$.

Le tableau II montre que pour tous les caractères soumis à la sélection, il y a soit stabilité, soit diminution de la variance génétique quand on passe de $P A_{0}$ à $P A_{1}$. La seule variance génétique qui augmente est celle du caractère $L A F$, caractère qui n'a pas été soumis à la sélection. Pour ce qui est de la variance résiduelle, toutes les situations existent. Les intervalles d'erreurs estimés sur les variances résiduelles montrent que pour aucune variable il n'est possible de rejeter au seuil de $5 \%$ l'hypothèse d'égalité de ces variances dans $P A_{0}$ et $P A_{1}$. Les héritabilités au 

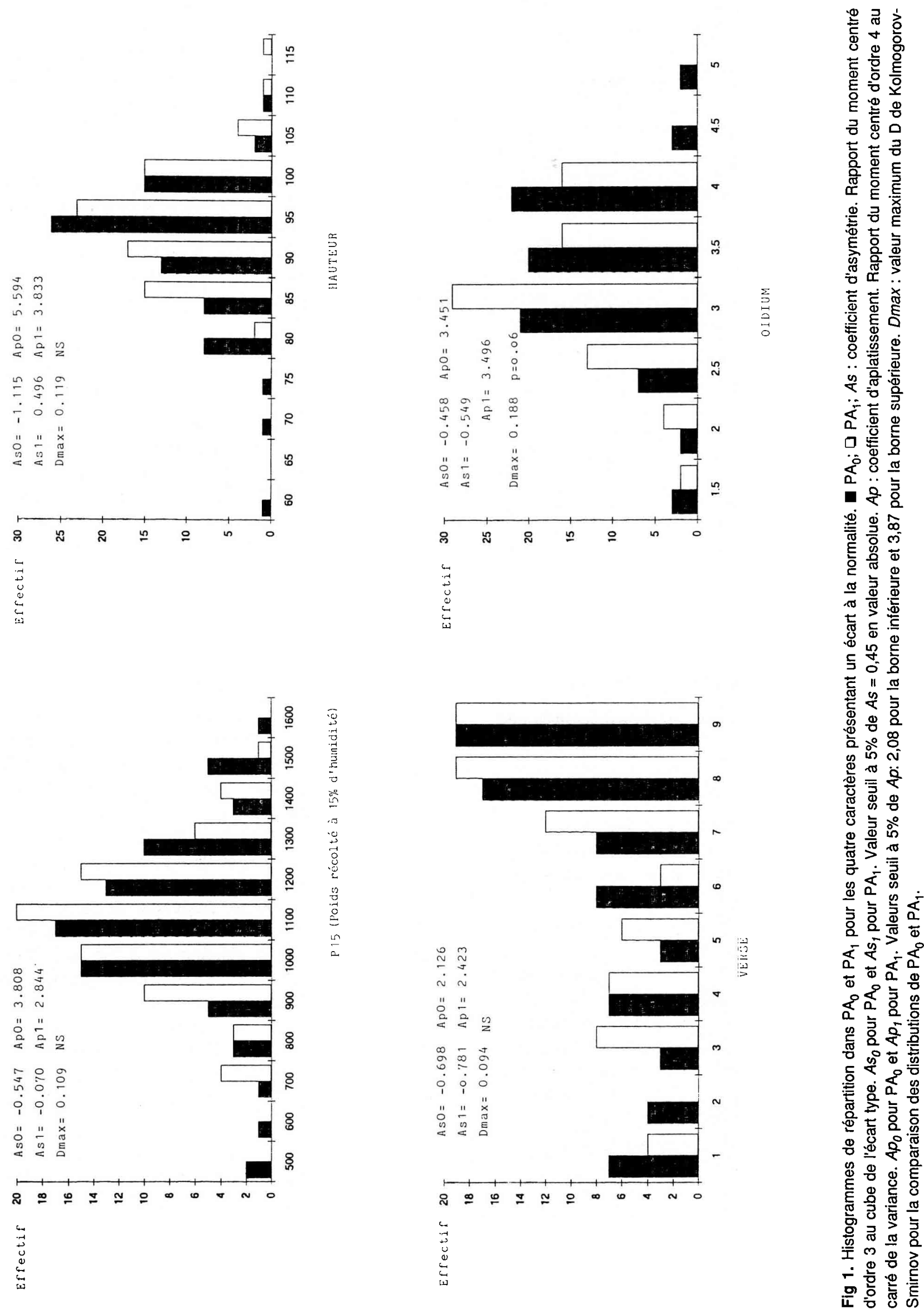
Tableau I. Comparaison de moyennes entre $\mathrm{PA}_{0}$ et $P A_{1}$. D\% obs : différence observée entre $P A_{1}$ et $P A_{0}$ en pourcentage de $\mathrm{PA}_{0}$ «/ indique que pour ce caractère le calcul n'est pas justifié. $D$ obs : différence observée entre $\mathrm{PA}_{1}$ et $\mathrm{PA}_{0}$. ${ }^{*}$ Significatif au seuil de $5 \%,{ }^{\star \star}$ significatif au seuil de $1 \%$. $D$ seuil $5 \%$ : différence attendue significative au seuil de $5 \%$ par le test de Student. Les signes indiquent si un test unilatéral ou bilatéral a été effectué. Pour les tests unilatéraux le signe indique le sens attendu de la déviation sous l'hypothèse d'un progrès.

\begin{tabular}{|c|c|c|c|c|c|}
\hline & $P A_{0}$ & $P A_{1}$ & $D \%$ obs & $D$ obs & $\begin{array}{c}D \text { seuil } \\
5 \%\end{array}$ \\
\hline$P 15$ & 1061 & 1031 & $-2,8$ & -30 & +29 \\
\hline$P C G$ & 3,98 & 4,07 & $+2,3$ & $+0,09^{*}$ & $+0,07$ \\
\hline P5E & 11,6 & 11,3 & $-2,6$ & $-0,3$ & $+0,3$ \\
\hline$H A U$ & 90,5 & 91,5 & $+1,1$ & +1 & $\pm 1,2$ \\
\hline VER & 6,15 & 6,35 & 1 & $+0,2$ & $-0,33$ \\
\hline$D E P$ & 33,5 & 33,2 & 1 & $-0,3$ & $\pm \quad 0,62$ \\
\hline LOF & 213,6 & 216,4 & $+1,3$ & $+2,8$ & $\pm 3,7$ \\
\hline$L A F$ & 17,7 & 17,7 & 0 & 0 & $\pm 0,25$ \\
\hline$N E E$ & 17,92 & 18,07 & $+0,8$ & $+0,15$ & $+\quad 0,18$ \\
\hline$O I D$ & 3,38 & 3,12 & $-7,6$ & $-0,26^{\star *}$ & $-0,18$ \\
\hline
\end{tabular}

P15: poids parcellaire ramené à $15 \%$ d'humidité, en g: $P C G$ : poids de cent grains, en g; $P 5 E$ : poids de cinq épis, en g; $H A U$ : hauteur au-dessus de l'épi en $\mathrm{cm}$; VER : verse, échelle de 1 à $9(1=$ absence...); $D E P$ : date d'épiaison en nombre de j après le $1^{\text {er }}$ mai; $L O N$ : Iongueur de la feuille de l'épi, en mm; $L A R$ : largeur de la feuille de l'épi, en mm; NEE : nombre d'épillets par épi; $O I D$ : note d'oïdium de 1 à 5 ( 1 = absence).

sens large estimées dans les 2 populations sont en général élevées. Les valeurs les plus faibles sont obtenues pour P5E dans les 2 populations et pour $P 15$ dans $P A_{1}$.

Le tableau II montre qu'il y a peu de corrélations génétiques fortes entre les caractères mesurés. On remarque 2 corrélations moyennes à fortes, positives et stables entre $P 15$ et $P 5 E$ et entre $H A U$ et VER. Les autres corrélations stables sont moyennes à faibles et traduisent des relations positives entre la hauteur et le poids de 100 grains, la date d'épiaison et le nombre d'épillets par épi, la longueur de la feuille de l'épi et la sensibilité à la verse, la précocité et le poids de 100 grains.

De nombreuses corrélations génétiques sont modifiées. On remarque toutefois que ces modifications n'affectent pas tous les caractères de la même façon. II n'y a en effet qu'une corrélation modifiée pour les 2 variables $P 15$ et $P C G$ et 2 pour P5E et LOF. La modification de corrélation entre P5E et LOF est la seule modification des relations entre ces 4 variables. Les variables $H A U, V E R, D E P, L A F$ et NEE voient par contre leurs interrelations nettement modifiées et sont aussi impliquées dans les modifications de corrélations avec les 4 variables précédemment $\mathrm{ci}$ tées.

La matrice des corrélations environnementales (tableau III) montre des corrélations en général proches de 0 . II existe 2 corrélations positives entre P5E et $P C G$ et entre $L O F$ et $L A F$ qui sont moyennes et stables et il y a 4 corrélations qui sont modifiées.

\section{DISCUSSION}

L'année 1987 a été très favorable à l'expression de la verse. Ceci explique en partie le coefficient de variation résiduel élevé que nous avons trouvé sur les témoins. L'écart à la normalité de la notation de verse est dû à des effectifs importants dans les classes de forte verse (fig 1). Les autres années, des écarts à la normalité sont constatés pour ce caractère pour la raison inverse. Pour la hauteur, un écart à la normalité était attendu puisque nous avions échantillonné dans la classe des lignées de taille inférieure à $1,10 \mathrm{~m}$.

La résistance à l'oïdium est sous contrôle de gènes majeurs, mais il existe aussi des résistances «quantitatives" qui s'expriment chez des variétés dépourvues de ces gènes (Lupton, 1987). La présence de gènes majeurs dans les parents fondateurs de la population PA n'est pas connue, par contre 3 géniteurs ont été introduits pour leur très bons comportements (Thomas, 1986). La dissymétrie droite qui apparaît dans $\mathrm{PA}_{1}$ (fig 1) et la comparaison de moyennes indiquent une sélection efficace qui a éliminé les individus les plus sensibles et enrichi la population en génotypes peu à moyennement sensibles. Nous trouvons sur ce caractère un progrès de $7,6 \%$. Un progrès de $16,2 \%$ sur la résistance à l'oïdium a aussi été trouvé par Thomas (1986) dans la comparaison des familles $S_{1}$ des 2 populations.

La dissymétrie observée pour le rendement dans la population $\mathrm{PA}_{0}$ pose quelques questions. Au-delà de l'hypothèse d'un simple effet de l'échantillonnage, on peut se demander si une liaison négative entre la hauteur et le rendement, plus forte dans $\mathrm{PA}_{0}$ que dans $\mathrm{PA}_{1}$, n'est pas à l'origine de ce résultat. En effet, sous cette hypothèse, le fait d'avoir gardé les lignées les plus 
Tableau II. Comparaison des variances et des héritabilités estimées dans $\mathrm{PA}_{0}$ et $\mathrm{PA}_{1}$. Var Gén : variance génétique estimée; $S g$ : écart type de l'erreur faite sur l'estimation de la variance génétique (Becker, 1984); $D \%$ : est égal à $\left(\left(\right.\right.$ Var Gén de $\left.P A_{1}\right)$ - (Var Gén de $\left.\left.P A_{0}\right)\right)$ / (Var Gén de $\left.P A_{0}\right) ;$ < Var Env < : variance environnementale estimée et intervalle de confiance à 5\%; H2 SL : héritabilité au sens large estimée par (Var Gén) / ((Var Gén) + (Var Env)).

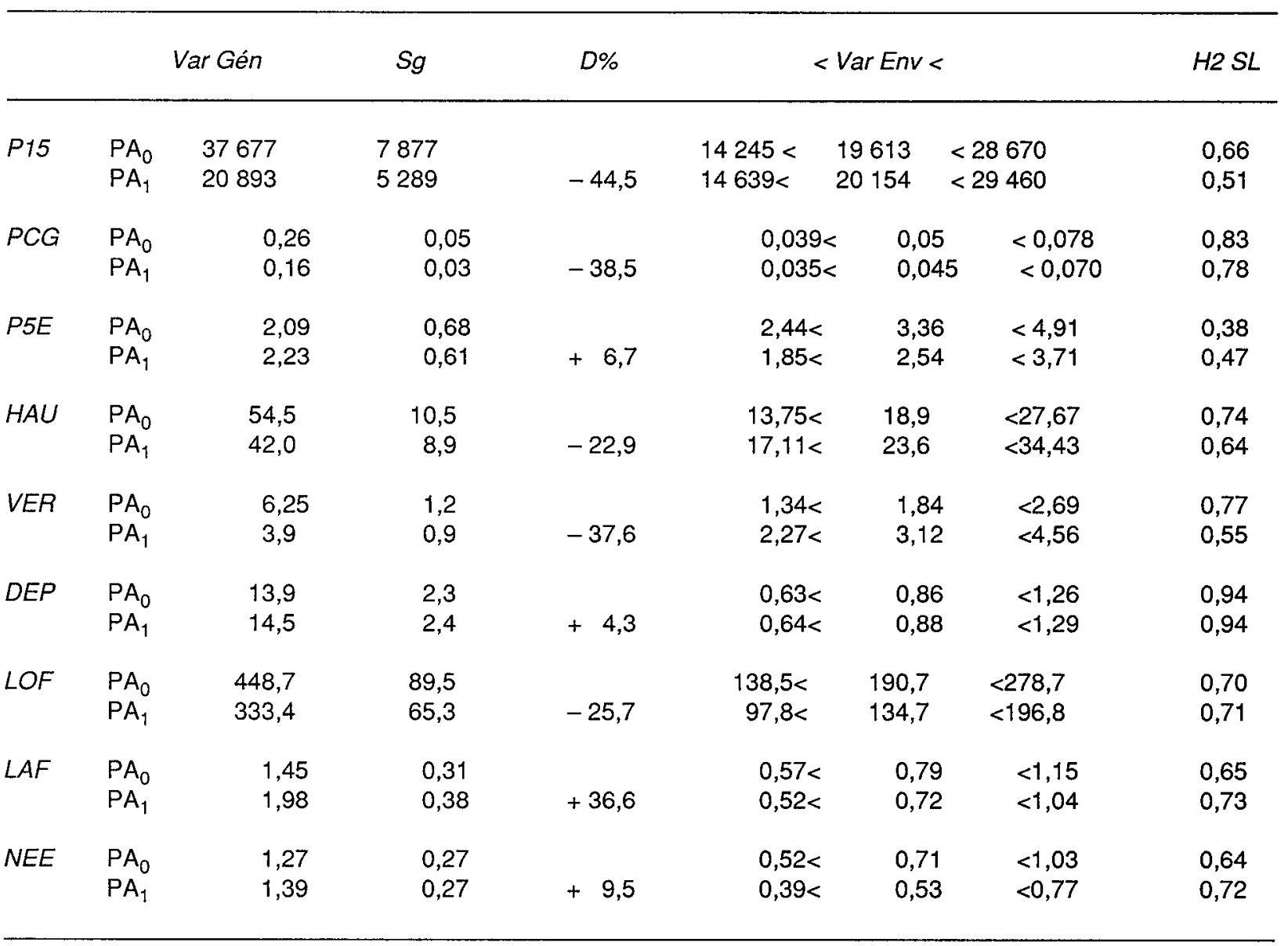

courtes aboutirait à enrichir la population $\mathrm{PA}_{0}$ en lignées moyennes à bonnes pour le rendement, comme nous le constatons. La corrélation phénotypique trouvée entre $P 15$ et $H A U$ est de 0,176 dans $\mathrm{PA}_{0}$ et de $-0,039$ dans $\mathrm{PA}_{1}$ (toutes 2 non significativement différentes de 0 ). Bien qu'estimées sur un échantillon qui ne représente pas toutes les hauteurs de chaque population, ces corrélations paraissent contredire l'hypothèse précédemment émise. Une autre explication peut être envisagée : $P A_{0}$ résulte du croisement pyramidal entre 16 lignées et il n'y a pas eu de cycle d'intercroisement à l'issue de ce plan de croisement. II n'est donc pas possible d'assimiler $\mathrm{PA}_{0}$ à une population panmictique et il est raisonnable de penser que d'importants déséquilibres de liaisons existent dans cette population. Ceci, en présence d'épistasie, peut expliquer des écarts à la normalité pour certains caractères quantitatifs.

La sélection sur le rendement ne paraît pas avoir été très efficace. Nous ne constatons aucun progrès sur ce caractère. Thomas (1986) constate sur les $\mathrm{S} 19$ que les $10 \%$ meilleures de $\mathrm{PA}_{1}$ sont moins bonnes que les $10 \%$ meilleures de $\mathrm{PA}_{0}$. Kervella (1987) trouve un progrès très limité allant de 0 à $4 \%$ suivant les méthodes d'estimation employées et les intervalles de confiances à $5 \%$ autour de ces estimations sont toujours chevauchants. Dans ce contexte, on peut penser que pour le rendement au moins, le premier cycle de sélection récurrente a consisté essentiellement en un intercroisement supplémentaire aboutissant à la normalisation constatée dans $\mathrm{PA}_{1}$.

L'absence de progrès constatée pour la hauteur et la résistance à la verse n'est pas surprenante étant donné l'échantillonnage que nous avons pratiqué. Nous n'attendions pas non plus de progrès sur la précocité puisqu'il n'y a pas eu de sélection sur ce caractère lors du premier cycle (Thomas, 1986). La variance génétique de ce caractère est restée constante. Kervella (1987) constate un gain d'une journée pour la 
Tableau III. Matrice des corrélations génétiques et environnementales. Comparaison entre $\mathrm{PA}_{0}$ et $\mathrm{PA}_{1}$. Corrélations environnementales au-dessus de la diagonale. Corrélations génétiques au-dessous. $0: P A_{0} ; 1: P A_{1}$.

\begin{tabular}{|c|c|c|c|c|c|c|c|c|c|c|}
\hline & & P15 & $P C G$ & P5E & $H A U$ & $V E R$ & $D E P$ & $L O F$ & $\angle A F$ & $N E E$ \\
\hline P15 & $\begin{array}{l}0 \\
1\end{array}$ & $\begin{array}{l}1 \\
1\end{array}$ & $\begin{array}{l}0,074 \\
0,106\end{array}$ & $\begin{array}{l}0,284 \\
0,124\end{array}$ & $\begin{array}{r}-0,259 \\
0,149\end{array}$ & $\begin{array}{l}-0,195 \\
-0,147\end{array}$ & $\begin{array}{r}-0,130 \\
0,277\end{array}$ & $\begin{array}{r}0,045 \\
-0,035\end{array}$ & $\begin{array}{r}0,009 \\
-0,169\end{array}$ & $\begin{array}{l}-0,169 \\
-0,013\end{array}$ \\
\hline PCG & $\begin{array}{l}0 \\
1\end{array}$ & $\begin{array}{l}0,125 \\
0,166\end{array}$ & $\begin{array}{l}1 \\
1\end{array}$ & $\begin{array}{l}0,410 \\
0,340\end{array}$ & $\begin{array}{r}0,122 \\
-0,019\end{array}$ & $\begin{array}{l}-0,032 \\
-0,161\end{array}$ & $\begin{array}{r}-0,031 \\
0,067\end{array}$ & $\begin{array}{r}-0,148 \\
0,036\end{array}$ & $\begin{array}{l}-0,045 \\
-0,018\end{array}$ & $\begin{array}{r}0,006 \\
-0,093\end{array}$ \\
\hline P5E & $\begin{array}{l}0 \\
1\end{array}$ & $\begin{array}{l}0,624 \\
0,503\end{array}$ & $\begin{array}{l}0,339 \\
0,159\end{array}$ & $\begin{array}{l}1 \\
1\end{array}$ & $\begin{array}{l}0,096 \\
0,222\end{array}$ & $\begin{array}{l}-0,014 \\
-0,153\end{array}$ & $\begin{array}{l}0,156 \\
0,170\end{array}$ & $\begin{array}{r}0,031 \\
-0,009\end{array}$ & $\begin{array}{r}0,056 \\
-0,078\end{array}$ & $\begin{array}{r}0,035 \\
-0,217\end{array}$ \\
\hline $\mathrm{HAU}$ & $\begin{array}{l}0 \\
1\end{array}$ & $\begin{array}{l}0,271 \\
-0,111\end{array}$ & $\begin{array}{l}0,402 \\
0,326\end{array}$ & $\begin{array}{r}0,012 \\
-0,122\end{array}$ & $\begin{array}{l}1 \\
1\end{array}$ & $\begin{array}{l}-0,075 \\
-0,202\end{array}$ & $\begin{array}{r}0,073 \\
-0,071\end{array}$ & $\begin{array}{l}-0,026 \\
-0,102\end{array}$ & $\begin{array}{r}0,144 \\
-0,002\end{array}$ & $\begin{array}{l}-0,004 \\
-0,082\end{array}$ \\
\hline VER & $\begin{array}{l}0 \\
1\end{array}$ & $\begin{array}{l}-0,259 \\
-0,141\end{array}$ & $\begin{array}{r}0,008 \\
-0,132\end{array}$ & $\begin{array}{l}-0,286 \\
-0,164\end{array}$ & $\begin{array}{l}0,445 \\
0,456\end{array}$ & $\begin{array}{l}1 \\
1\end{array}$ & $\begin{array}{r}0,082 \\
-0,012\end{array}$ & $\begin{array}{r}0,092 \\
-0,095\end{array}$ & $\begin{array}{l}-0,120 \\
-0,130\end{array}$ & $\begin{array}{l}0,127 \\
0,167\end{array}$ \\
\hline DEP & $\begin{array}{l}0 \\
1\end{array}$ & $\begin{array}{l}-0,038 \\
-0,138\end{array}$ & $\mid \begin{array}{l}-0,400 \\
-0,256\end{array}$ & $\begin{array}{l}-0,132 \\
-0,087\end{array}$ & $\begin{array}{r}-0,261 \\
0,154\end{array}$ & $\begin{array}{l}0,046 \\
0,323\end{array}$ & $\begin{array}{l}1 \\
1\end{array}$ & $\begin{array}{l}0,067 \\
0,412\end{array}$ & $\begin{array}{r}0,062 \\
-0,095\end{array}$ & $\begin{array}{l}-0,192 \\
-0,046\end{array}$ \\
\hline LOF & $\begin{array}{l}0 \\
1\end{array}$ & $\begin{array}{r}-0,096 \\
0,030\end{array}$ & $\begin{array}{l}0,101 \\
0,062\end{array}$ & $\begin{array}{r}-0,014 \\
0,460\end{array}$ & $\begin{array}{l}0,185 \\
0,516\end{array}$ & $\begin{array}{l}0,217 \\
0,371\end{array}$ & $\begin{array}{l}0,160 \\
0,180\end{array}$ & $\begin{array}{l}1 \\
1\end{array}$ & $\begin{array}{l}0,432 \\
0,307\end{array}$ & $\begin{array}{l}-0,15 \\
-0,125\end{array}$ \\
\hline LAF & $\begin{array}{l}0 \\
1\end{array}$ & $\begin{array}{l}-0,154 \\
-0,048\end{array}$ & $\begin{array}{l}0,390 \\
1 \quad 0,062\end{array}$ & $\begin{array}{l}0,185 \\
0,260\end{array}$ & $\begin{array}{r}-0,327 \\
0,001\end{array}$ & $\begin{array}{r}-0,016 \\
0,196\end{array}$ & $\begin{array}{l}0,167 \\
0,520\end{array}$ & $\begin{array}{l}0,143 \\
0,230\end{array}$ & $\begin{array}{l}1 \\
1\end{array}$ & $\begin{array}{l}-0,025 \\
-0,110\end{array}$ \\
\hline NEE & $\begin{array}{l}0 \\
1\end{array}$ & $\begin{array}{l}-0,054 \\
-0,067\end{array}$ & $\begin{array}{l}-0,102 \\
-0,207\end{array}$ & $\begin{array}{l}0,347 \\
0,117\end{array}$ & $\begin{array}{r}-0,378 \\
0,074\end{array}$ & $\begin{array}{r}-0,296 \\
0,020\end{array}$ & $\begin{array}{l}0,264 \\
0,334\end{array}$ & $\begin{array}{l}0,106 \\
0,287\end{array}$ & $\begin{array}{l}0,272 \\
0,018\end{array}$ & $\begin{array}{l}1 \\
1\end{array}$ \\
\hline
\end{tabular}

Corrélations fortes ou moyennes et stables; i Corrélations modifiées.

précocité mais ne constate pas non plus de modification de la variance du caractère. Pour le poids moyen d'un épi (mesuré par P5E dans cette étude), Thomas (1986) constate comme nous une légère régression (test de signification non effectué), alors que Kervella (1987) trouve une légère progression (non significative à $5 \%$ ). Pour ce caractère on peut donc penser qu'il n'y a pas eu de progrès. Pour le poids de 100 grains (poids de 1000 grains dans les 2 autres études), les 3 études montrent un progrès. Pour le nombre d'épillets par épi, Thomas (1986) trouve un progrès de $8,5 \%$ alors que nous ne constatons aucun progrès.

Quand on compare $P A_{0}$ et $P A_{1}$, il existe une évolution à la baisse de certaines variances génétiques qui a aussi été constatée dans les 2 études déjà citées. La sélection et l'intercroisement supplémentaire ont donc eu une influence sur la variabilité. De ce point de vue, l'évolution des corrélations entre caractères est intéressante à observer. Les caractères liés au développement, hauteur, date d'épiaison, largeur de la feuille de l'épi et nombre d'épillets par épi voient leurs interrelations nettement perturbées. Pour les autres caractères peu de modifications sont constatées.

La matrice des corrélations environnementales montre des corrélations en général proches de 0 et une grande stabilité. Cette stabilité n'est pas surprenante étant donné que les lignées des 2 populations ont été expérimentées dans les mêmes conditions et qu'il existe un fort apparentement entre $\mathrm{PA}_{0}$ et $\mathrm{PA}_{1}$.

L'absence de progrès sur le rendement montre que bien qu'on estime dans la population PA une forte héritabilité au sens large (tableau II, Brabant et al, 1989), l'héritabilité au sens étroit doit être faible. De plus, la sélection sur le rendement a été multilocale et multicaractère. Ceci 
peut aussi expliquer des gains faibles dans chaque lieu et sur un caractère particulier entrant dans l'index. II serait intéressant de juger dans une expérimentation multilocale s'il y a eu gain en stabilité pour le rendement. Pour les caractères quantitatifs, il est possible de déterminer les coefficients à leur appliquer dans un index, grâce au contrôle a priori des progrès attendus sur chacun d'entre eux (Sampoux, 1989). L'abandon du système empirique de pondération sera sans doute une source de plus grande efficacité.

Pour améliorer la prédiction du rendement en sélection précoce, il est possible d'utiliser les corrélations avec des caractères associés. Les caractères $L O F$ et $L A F$ ont été étudiés pour juger s'il existait une corrélation intéressante avec le rendement. Comme nous le constatons dans le tableau III, la seule corrélation génétique forte avec P15 est celle de P5E. Pour les autres caractères aucune corrélation ne paraît utilisable. Cette conclusion est tout à fait cohérente avec celle de l'étude faite sur les $S_{1}$ du troisième cycle de sélection récurrente appliquée à la population PA (Brabant et al, 1989).

\section{CONCLUSION}

L'étude présentée ici indique que pour un caractère peu héritable (au sens de la transmission après intercroisement) comme le rendement, if est très difficile de juger du sens de son évolution après un seul cycle de sélection récurrente multicaractère. L'évolution à la baisse de la variance génétique constatée pour ce caractère, n'est sans doute pas affectable à une perte de variabilité en terme de richesse allélique mais bien plus certainement à une réorganisation de la variabilité. Pour expliquer cette réduction on peut évoquer, comme le font Kervella et al (1988) suivant le modèle de Bulmer (1976), des déséquilibres de liaison créés par la sélection qui génère un excès d'associations en «répulsion" et un écart de la panmixie dû aux options prises lors de l'intercroisement (ne pas croiser des familles ayant les mêmes défauts). Nous pensons qu'il est aussi possible d'évoquer le rôle de l'intercroisement supplémentaire pour réduire des déséquilibres de liaison initiaux existant dans $P A_{0}$. En effet, un excès de situations en "couplage» peut expliquer l'aplatissement de la distribution observée dans $\mathrm{PA}_{0}$. Dans les 2 cas on ne s'attend pas à ce que la tendance à la réduction constatée lors du premier cycle se maintienne avec la même intensité au cours des cycles suivants. Un contrôle de cette évolution paraît toutefois souhaitable.

Les études déjà menées sur la comparaison des 2 premiers cycles de sélection récurrente appliqués à PA (Thomas, 1986; Kervella, 1987; Kervella et al, 1988 et Rousset et al, 1988) montrent que la sélection multicaractère a engendré un progrès sur la résistance aux maladies et sur la qualité boulangère. II apparaît donc nécessaire de mieux juger des potentialités de rendement des familles soumises à la sélection précoce et de revoir les modalités de constitution de l'index pour assurer aussi un progrès significatif sur le rendement.

\section{RÉFÉRENCES}

Bachacou J, Masson JP, Millier C (1981) Manuel de la programmathèque statistique Amance. INRA

Becker WA (1984) Manual of quantitative genetics. Academic entreprises, Pullman, Washington

Bulmer MG (1976) The effect of selection on genetic variability: a simulation study. Genet Res 28,101 117

Brabant P, Manes Y, Trottet M, Picard E (1989) Corrélations génétiques, héritabilités et possibilités de sélection multilocale précoce sur le rendement chez le blé tendre d'hiver (Triticum aestivum L). Agronomie 9, 49-54

Fouilloux G, Bannerot $H$ (1988) Selection in the common bean (Phaseolus vulgaris). P Gepts Kluwer Acad Publ, Boston, London, 503-542

Gallais (1970) Covariances entre apparentés quelconques avec linkage et épistasie. I. Expression générale. Ann Génét Sél Anim 2, 417-427

Gallais A (1977) Amélioration des populations, méthodes de sélection et création de variétés. I. Synthèse critique sur les problèmes généraux et sur les bases théoriques pour la sélection récurrente intrapopulation. Ann Amélior Plant 27, 281-330

Gallais A (1978) Amélioration des populations, méthodes de sélection et de création de variétés. II. Le concept de valeur variétale et ses conséquences pour la sélection récurrente. Ann Amélior Plant 28, 269-287

Gallais A (1979) Le concept de valeur en lignées d'un génotype et son utilisation possible en sélection. Ann Amélior Plant 29, 1-22

Gallais A (1988) A method of line development using doubled haploids: the single haploid descent recurrent selection. Theor Appl Genet 75, 330-332

Goulden $\mathrm{CH}$ (1939) Problems in plant selection. Proc 7th Int Genet Cong Edimburgh (RC Punnet, ed) Cambridge Univ Press, 132-133 
Hull FH (1945) Recurrent selection for specific combining ability in corn. J Am Soc Agron 37, 134-135

Kervella J (1987) Sélection récurrente, chez le blé : bilan d'un cycle. Thèse de doctorat de l'université de Paris-Sud Orsay

Kervella J, Rousset M, Doussinault G, Picard E (1988) Efficiency of a first cycle recurrent selection for variety development in Wheat. Proc 7 th Meeting Eucarpia Section Biometrics in Plant Breeding. Norwegian St Agric Res St, Norway, 14-22

Lupton FGH (1987) Wheat breeding. Its scientific basis. Chapman and Hall, Londres, New York
Rousset M, Kervella J, Pichon M, Branlard G, Doussinault $G$, Picard $E$ (1988) Effectiveness of recurrent selection for bread making quality breeding in wheat (Triticum aestivum $L$ em Thell) Proc 7 th Int wheat genetic Symposium (Miller TE, Koebner RMD, eds) Cambridge, UK, 1009-1023

Sampoux JP (1989) L'index de sélection sur plusieurs caractères : une synthèse sur les pondérations économiques, le choix de contraintes et de caractères associés. Agronomie 9, 993-999

Thomas G (1986) Étude d'une population de blé tendre (Triticum aestivum $L$ ) en sélection récurrente à cycle court. Thèse de doctorat univ Rennes 